The results already obtained have modified and corrected many of our former ideas concerning the nature of the initial phase of slow uniform flame-movement in gaseous explosions, and (inter alia) completely disproved the supposed ' law of flame speeds '. Indeed, it now seems probable that what Mallard and Le Chatelier visualised as flame propagation 'by conduction 'is an ideal condition perhaps realisable only when a stagnant explosive mixture is ignited without impulse at the centre of a spherical vessel of infinite radius.

Important new information has been obtained regarding the influences of 'compression' and 'shock' waves upon the speeding up of combustion and flame movements during explosions ; and it is now proved that the speed can be abruptly raised from a lower to a higher uniform value when a flame is overtaken by a 'shock' wave travelling in the same direction. Indeed, it has been shown that in such manner the flame-speed can be successively raised per saltum many times, and that it may assume any uniform value between the limits of that theoretically corresponding with propagation by 'conduction' and that due to propagation 'by adiabatic compression'.

Much new light has been thrown on what may be termed the 'pre-detonation' phase of explosion, when the flame is advancing at a speed greater than that of any 'shock' wave through the unburnt medium, and is therefore overtaking any such shock waves which are ahead of it. 'Detonation' is ultimately set up when a rapidly moving flame on the verge thereof is just about to overtake a shock wave immediately in front of it. Thereupon an 'ignition ahead' of the flame-front occurs, and immediately afterwards ' detonation' is set up. Indeed, during the 'pre-detonation' phase a series of successive such 'ignitions ahead' may occur, detonation being set up immediately after the last one.

We have obtained photographs analysing the phenomenon of 'spin' in detonation, which was first observed five years ago in detonations of moist $2 \mathrm{CO}+\mathrm{O}_{2}$ mixtures by C. Campbell and D. W. Woodhead, working in the late H. B. Dixon's laboratory, and later confirmed in our laboratories at South Ken. sington. 'Spin' has also been observed in detonations of methane-oxygen, pentane-oxygen, undiluted acetylene, and other media, and apparently is caused by the helical rotation of a luminous 'head' of detonation in the flame front together with a long luminous "tail" behind it. The pitch $(L)$ and frequency $(f)$ of such rotation in detonations of a given medium varies with the internal diameter $(D)$ of the tube, so that, while the ratio $L / D$ for diameters up to about 1 inch is constant, and nearly equal to $3 \cdot 0$, the helical velocity of the rotating ' head' of detonation is approximately of the same order, irrespective of the medium or of the tube diameter. Thus, in the case of a moist $2 \mathrm{CO}+\mathrm{O}_{2}$ medium, which exhibits the phenomenon most markedly, $f=44,000$ per sec., and $L=3.95 \mathrm{~cm}$. in a tube of $1.3 \mathrm{~cm}$. internal diameter, the velocity of the 'head' of detonation along its helical path being 2500 metres per second.

These discoveries have necessitated revision of the old classic conception of 'detonation' and opened up new lines of inquiry concerning it.

Within recent years the photographic method has also been used for the purpose of analysing (i) ignition phenomena, including the 'induction' period of explosions, (ii) the influence of moisture upon explosions of carbonic oxide-oxygen mixtures, and (iii) the influence of strong electrical fields upon gaseous explosions.

\title{
The Construction of Man's Family Tree.*
}

SOMEWHERE about the year 1865 Ernest Haeckel drew a family tree to represent man's relationship to the rest of the animal kingdom. Neither Darwin nor Huxley were so daring. In 1871 Darwin went no further in his "Descent of Man " than to infer that "a member of the anthropomorphous subgroup gave birth to man". In 1863 the utmost Huxley adventured was the opinion that man had arisen by "the gradual modification of a man-like ape or from the same stem ". Haeckel saw life as a great tree rooted deeply in the geological past with only the end-twigs peering through the surface of the earth into the present as living forms. The terminal twig on the extreme right of his tree represents humanity (Homo sapiens). Man, the gorilla, and the chimpanzee are made to emerge from the same terminal stem. Following this human-gorilla stem to the left downwards, we find shooting from it the orang twig, then at a still longer interval, the gibbon branch, then the old world monkeys, then those of the new world, and ultimately this primate stem merges with the stem of the prosimiæe or lemurs.

The facts which guided Haeckel were the multitude of structural points in common which could be explained only as an inheritance from a common ancestor. To convert his hypothesis into true history it was necessary to find the fossilised remains of his missing links ; but very few of these were known in his day-Neanderthal man and Dryopithecus and Pliopithecus from the Miocene deposits of Europe. Haeckel depicted the human stem as breaking away from that leading on to the gorilla and the chimpanzee

* Substance of an evening discourse delivered before the British Association on Sept. 26 by Sir Arthur Keith, F.R.S. rather more than half-way through the Tertiary period. On Osborn's estimation in years, Haeckel thus gives man's antiquity at 20 or 25 millions of years.

In 1895 Dr. Eugen Dubois, in order to incorporate his newly discovered Pithecanthropus, showed the central stem as represented at first by a procero. pithecus, the ancestor of the old world monkeys, then the prothylobates, the ancestor of the gibbons, then the prototype of the great anthropoid apes, followed by the Pithecanthropus type and finally the stem ending in the human form.

Some years previously Sir Arthur Keith was en. gaged with the dissection of some 300 specimens, gorillas, chimpanzees, etc., and the collection of records of human dissections, in the hope of arriving at a clear-cut explanation of the distribution of anatomical characters. In 1900 he drafted a chart to show the mass of data thus collected. He arrived at the conclusion that the evolution of posture gives the clue not only to the evolution of man but also to that of all the higher primates. There are only two main ways in which an animal can climb a tree-either with the body horizontal (pronograde) or upright (orthograde). Sir Arthur Keith concluded that an arboreal orthograde anthropoid had first diverged in a humanward direction in specialisation of spine, leg, and foot. It was on the trees and not on the ground that man first came by the initial stages of his posture and carriage.

What has happened in the last thirty years confirms the conviction that in the study of the gibbon is the key to the evolution of the great anthropoids and man. In 1910 the discovery of Propliopithecus in the older

No. 3231, VoL. 128] 
oligocene of Egypt extended the antiquity of what is apparently an ancestor of the gibbons, and presumably orthograde, for a good many million years. It affords a fixed point for speculation concerning the evolution of the orthograde posture. Prof. Osborn and Prof. Wood Jones, who exclude the other anthropoids from the ancestry of man, are prepared to accept Propliopithecus as an ancestor from which both man and the chimpanzee may have arisen.

Sir Arthur Keith's census showed so many anatomical characters in common in the big-bodied group, gorilla, chimpanzee, and orang, that he postulated a common ancestor for the big-bodied group. The discoveries of Dr. Foutan in the lower Miocene of Egypt (1920) of two orthograde primates, one the size of a gibbon, the other half-way between the gibbon and man, support his contention that the great orthograde primates evolved at the beginning of the Miocene. It was at this period, evidently one of great plasticity, that Sir Arthur Keith supposes the human line to have separated from the great anthropoids. Dr. W. K. Gregory also brings the human stem from the anthropoids at the beginning of the Miocene. They both give the human stem an antiquity of about 20 million years.

The human posture was completely evolved before the end of the pliocene period. Allowing two geological periods, the Miocene and the Pliocene, this represents about 18 million years for the evolution of the human characteristics of foot, leg, thigh, and pelvis. Beside Pithecanthropus we now know two other forms of early Pleistocene man, Eoanthropus and Sinanthropus. By the end of the Pleistocene, then, the human stem had been in existence long enough to have broken up into many divergent branches; but we are uncertain whether they represent the ancestors of any race now living. Hence in our tree we trace the living races to an early pleistocene ancestor not yet discovered.

It is not claimed that this formula explains all the facts. Prof. Wood Jones points out that the human body preserves many primitive features which have disappeared from those of anthropoids and of monkeys--features which Sir Arthur Keith thinks are to be explained not by modifying the family tree but by a fuller understanding of the laws of inheritance. In the United States, Dr. W. K. Gregory assigns a place to Australopithecus, the Taungs skull, nearest to man. Dr. A. H. Schultz, of Johns Hopkins University, has drafted a tree in which the gibbon and man are opposed as contrasted forms, while Prof. H. Fairfield Osborn excludes the anthropoidal stage from human ancestry.

Sir Arthur Keith concludes by giving his support to the form of man's family tree which was drafted by Haeckel sixty-five years ago. All are agreed that anthropoid and man have a common ancestry; it is merely the degree of relationship which is in dispute.

\section{A Retrospect of Wireless Communication.*}

SIR OLIVER LODGE said that, looking back over his connexion with wireless telegraphy, the first paper which had attracted his attention was one by Lord Kelvin in 1853 on "Transient Currents", which gave the theory of electric oscillations in a masterly manner considering that the idea of self-induction was not then born. Kelvin knew that electric energy could be stored in and released by a condenser in a manner analogous to that in which energy could be stored and released by a coiled spring, and, moreover, that the current resulting from the condenser discharge possessed properties akin to inertia or momentum. The discharge would consequently be of an oscillating character unless the circuit resistance exceeded a certain critical value, and the resulting spark a beaded band instead of a continuous band.

With a large enough condenser, oscillations should be slow enough to give a musical note. The frequency of the note depends on the capacity of the circuit and on its self-induction, which Kelvin spoke of as the "electrodynamic capacity of the discharging circuit". Such a musical note was exhibited by Sir Oliver at the Royal Institution in what was called a "whistling spark', the pitch of which could be brought down to reach the tones of the human voice.

One factor the theory did not take into consideration was possible loss of energy by radiation, in the same way as a tuning-fork mounted on a soundingboard loses energy to the surrounding air, thus diminishing the time during which the charge would oscillate. At the time, such radiation was unsuspected, and in any case there were no means of detect. ing it.

The first to show that an electric discharge would generate radiation was Fitzgerald, who discussed the problem mathematically in a paper read before the British Association in 1880. In a further paper in 1883 , he calculated the actual energy lost per second by a given condenser and self-induction and showed

* Substance of an evening discourse delivered before the British Association on Sept. 26 by Sir Oliver Lodge. that it was proportional to the fourth power of the frequency. To obtain an efficient radiator it was necessary to work with a frequency of not less than one million cycles. Fitzgerald took as the basis of his research the results of Maxwell's investigations on electromagnetic theory

In 1865, Clerk Maxwell had attempted to treat mathematically what Faraday had long brooded over as the 'electric field', regarding it as distinct from matter and existing in the ether of space round the charged body. Maxwell evolved two sets of equations, one for the electrostatic field and another for the magnetic field surrounding a current. These he combined in an endeavour to discover the nature of the electromagnetic field. His result, which is now familiar to scientific workers, was the differential equation expressing wave motion. Fortunately, the separate electric and magnetic constants of the ether, which are immeasurable, did not enter into the expression except as a product, which experiments in Germany had shown to be capable of determination.

Maxwell set to work to determine experimentally the rate of propagation of the electromagnetic waves predicted by his theory, and obtained the surprising result that it was equal to the velocity of light.

Theories of light up to this time had treated the ether as an elastic solid capable of transmitting vibrations at a tremendous speed. No single theory had been able to account for all the phenomena of light, and Maxwell's theory, published in 1873 and representing a complete departure from accepted ideas, came as a revelation. His equations have formed the basis of all subsequent work on electromagnetic waves.

Attempts were soon made to verify Maxwell's theory experimentally by the production of ether waves by electro-magnetic means. In 1887 or 1888, Sir Oliver Lodge, working with closed condensers, obtained evidence of their existence in the form of nodes or loops characteristic of ether waves reflected back on them. selves at the end of a wire attached to such condensers.

No. 3231, VoL. 128] 